\title{
Efficacy of moderately hypofractionated simultaneous integrated boost intensity- modulated radiotherapy combined with temozolomide for the postoperative treatment of glioblastoma multiforme: a single-institution experience
}

Liangzhi Zhong ${ }^{1 \dagger}$, Lu Chen ${ }^{1+}$, Shengqing Lv², Qingrui Li $i^{3}$, Guangpeng Chen ${ }^{1}$, Wen Luo ${ }^{1}$, Pu Zhou ${ }^{1}$ and Guanghui Li ${ }^{i^{*}}$ (D)

\begin{abstract}
Purpose: Despite recent advances in multimodal treatments, the prognosis of patients with glioblastoma multiforme (GBM) remains poor. The aim of this study was to evaluate the efficacy of moderately hypofractionated simultaneous integrated boost intensity-modulated radiotherapy (SIB-IMRT) combined with temozolomide (TMZ) for the postoperative treatment of GBM.

Materials and methods: From February 2012 to February 2018, 80 patients with newly diagnosed and histologically confirmed GBM in our institute were reviewed retrospectively. All patients underwent complete resection or partial resection surgery and then received hypofractionated SIB-IMRT with concomitant TMZ followed by adjuvant TMZ. A total dose of 64 Gy over 27 fractions was delivered to the gross tumor volume (GTV), clinical target volume 1 (CTV1) received 60 Gy over 27 fractions, and CTV2 received 54 Gy over 27 fractions. The progression-free survival (PFS) and overall survival (OS) rates and the toxicities were evaluated. Prognostic factors were analyzed using univariate and multivariate Cox models.
\end{abstract}

Results: The median follow-up was 16 months (range, 5 72 months). The median PFS was 15 months, and the 1-, 2-, and 3-year PFS rates were 56.0, 27.6, and 19.5\%, respectively. The median OS was 21 months, and the 1-, 2-, 3-, and 5-year OS rates were $77.6,41.6,32.8$, and $13.4 \%$, respectively. The toxicities were mild and acceptable. Age, KPS scores and the total number of TMZ cycles were significant factors influencing patient survival.

Conclusion: Moderately hypofractionated SIB-IMRT combined with TMZ is a feasible and safe treatment option with mild toxicity and good PFS and OS.

Keywords: Glioblastoma multiforme, Simultaneous integrated boost intensity-modulated radiotherapy, Temozolomide, Toxicity, Efficacy

\footnotetext{
*Correspondence: liguanghui_2000@aliyun.com

'Liangzhi Zhong and Lu Chen contributed equally to this work and should be considered co-first authors

${ }^{1}$ Cancer Research Institute of the Chinese People's Liberation Army, Xinqiao

Hospital, Army Medical University, Chongqing 400037, China

Full list of author information is available at the end of the article
}

(c) The Author(s). 2019 Open Access This article is distributed under the terms of the Creative Commons Attribution 4.0 International License (http://creativecommons.org/licenses/by/4.0/), which permits unrestricted use, distribution, and reproduction in any medium, provided you give appropriate credit to the original author(s) and the source, provide a link to the Creative Commons license, and indicate if changes were made. The Creative Commons Public Domain Dedication waiver (http://creativecommons.org/publicdomain/zero/1.0/) applies to the data made available in this article, unless otherwise stated. 


\section{Background}

Glioblastoma multiforme (GBM) is the most common and aggressive malignant brain tumor in adults. The current standard of care for newly diagnosed GBM is maximal surgical resection followed by radiotherapy (RT) in association with concomitant and adjuvant temozolomide (TMZ); currently; the median OS is 14.6 months [1]. Despite advances in microsurgical techniques, RT and chemotherapy, prognosis remains poor, and more than $90 \%$ of patients die within 5 years. The pattern of failure is primarily tumor recurrence. Studies have shown that approximately $90 \%$ of recurrence occurs within the original treatment field [2-5], which could result from insufficient therapeutic doses [6]. Therefore, high-dose radiation seems reasonable to improve local control with the hope of improving survival.

Dose escalation can be achieved by increasing the total dose or the dose per fraction. Increasing the total dose seems logical, but a previous study suggested that highdose conformal RT did not improve survival despite the total dose delivered being up to 90 Gy [7]. Hypofractionation is an alternative method to increase the biological effect of RT, which can achieve increased cell kill from a higher dose per fraction and offset the acceleration of tumor cell repopulation by shortening the overall treatment time [8]. However, the larger the dose per fraction delivered, the higher the rate of radionecrosis is $[9,10]$, which may hinder the application of hypofractionated $\mathrm{RT}$. To reduce the maximum dose to the organs at risk and increase the maximum dose to the target volumes, the simultaneous integrated boost intensity-modulated radiotherapy (SIB-IMRT) technique was developed [11].

Previous studies have investigated the efficacy and safety of hypofractionated SIB-IMRT combined with TMZ [12-14]. In 2012, we began to use moderately hypofractionated SIB-IMRT combined with concomitant and adjuvant TMZ in postoperative patients with GBM. The aim of the current study is to report on the efficacy of this regimen, including treatment-related toxicity, local recurrence, progression-free survival (PFS), and overall survival (OS).

\section{Materials and methods}

Patient population

From February 2012 to February 2018, a total of 526 patients were diagnosed with glioma in our hospital. Among these patients, 207 were diagnosed with highgrade glioma. Patients who had histologically confirmed GBM (World Health Organization [WHO] grade IV astrocytoma) were included in this study. Patients with GBM had undergone surgical resection before RT in our institution. Therefore, this study included only postoperative patients. No limitations were placed on Karnofsky performance status (KPS), age, lesion location or extent of surgery. This was a retrospective analysis, and approval was obtained from the institutional review board and ethics committee.

\section{Treatment planning and delivery}

RT was initiated as soon as possible after surgery, within 6 weeks in most cases. Patients were placed in supine position with a customized immobilization device. Each patient underwent a spiral computed tomography (CT) scan with $3 \mathrm{~mm}$ slice thickness. The $\mathrm{CT}$ images were then transferred to and registered in the treatment planning system (Varian Medical System, Palo Alto, USA). The gross tumor volume (GTV) was defined as all contrast-enhancing lesions on the postoperative MRI T1-weighted images and the postoperative cavity with the latter fused with computed tomography images for treatment planning. The clinical target volume (CTV) 1 was defined as GTV plus a 1 2-cm margin, including surrounding edema on T2-weighted fluid-attenuated inversion recovery MRI. If the edema was beyond $2 \mathrm{~cm}$ from the GTV, the margin was expanded to include all of the area exhibiting edema. CTV2 was defined as CTV1 plus a $1 \sim 2-\mathrm{cm}$ margin. The planning target volume (PTV), including PGTV, PCTV1, and PCTV2, was defined as the respective above target volume plus a $0.3-\mathrm{cm}$ margin. The margin could be modified to a smaller margin if there were organs at risk (OARs), such as the brain stem, optical pathway, or spinal cord, or if there were anatomical barriers, such as the dura, tentorium, and falx cerebri.

Treatment using a step-and-shoot technique with 6 MV photon beams was delivered with an MLC-equipped megavoltage linear accelerator (Trilogy Linac, Varian, USA). Daily doses of 2.4 Gy, 2.2 Gy, and 2.0 Gy were delivered to PGTV, PCTV1, and PCTV2 with a total dose of $64 \mathrm{~Gy}, 60 \mathrm{~Gy}$ and $54 \mathrm{~Gy}$, respectively, in 27 fractions over 6 weeks. The planning goal was the prescription dose encompassing at least $95 \%$ of the PTV, and no more than $10 \%$ of the PTV received more than $110 \%$ of the prescribed dose. The critical structures included the brain stem, optic chiasm, lens, optic nerves, and hippocampus. The upper dose constraints for the brain stem, optic nerves and chiasm are $54 \mathrm{~Gy}$, the maximum for lenses is $5 \mathrm{~Gy}$, the maximum dose for hippocampus is less than $24 \mathrm{~Gy}$, and the mean dose was less than $12 \mathrm{~Gy}$. The targets and dose distribution in a representative patient are shown in Fig. 1.

All patients except for 5 patients, due to personal reasons, received concomitant TMZ $\left(75 \mathrm{mg} / \mathrm{m}^{2} /\right.$ day) every day during RT. After a 4-week break, the patients received adjuvant TMZ (150-200 $\mathrm{mg} / \mathrm{m}^{2} /$ day) for 5 days every 28 days. The total number of TMZ cycles were determined by oncologists according to the patients' 


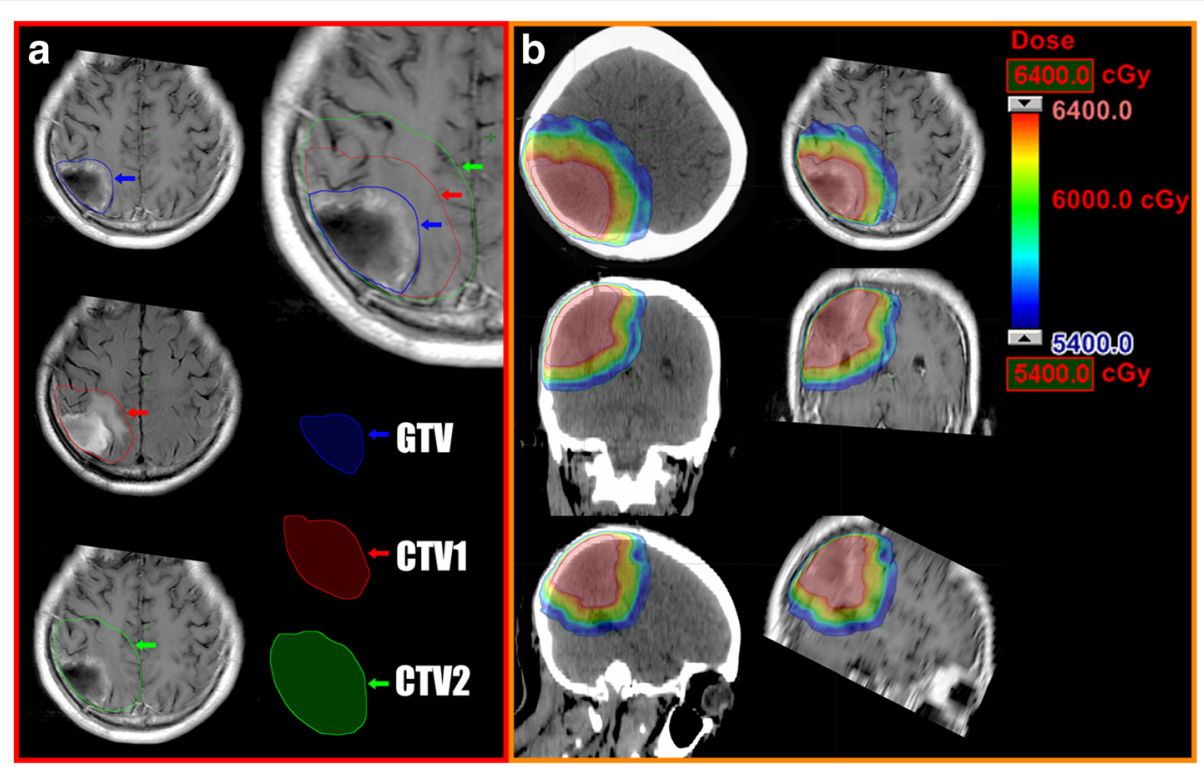

Fig. 1 The target volume delineation and isodose distribution of a representative patient who received hypofractionated SIB-IMRT. a GTV is identified with a blue arrow, including the contrast-enhanced lesion on the postoperative T1-weighted MR images and the postoperative cavity; CTV1 is identified with a red arrow, including GTV plus a 1-cm margin and the surrounding edema on T2-weighted fluid-attenuated inversion recovery MRl; CTV2 is identified with a blue arrow, including CTV1 plus a 2-cm margin. b Isodose distribution in CT and MRI, showing the target volumes receiving 64 Gy (red), 60 Gy (green), or 54 Gy (blue)

general condition, compliance, economic situation and disease progression.

\section{Follow-up}

Patients were followed weekly during the treatment period, with a medical history, physical examination, and complete blood test, and then patients were followed every month after finishing the treatment for at least 3 months. All patients were followed routinely with neurological examinations and MRI imaging at 3- to 6-month intervals after the treatment. MR spectroscopy and MR perfusion of the brain were not routinely used, except when in doubt about tumor progression or necrosis. Acute toxicities were scored using the Common Terminology Criteria for Adverse Events, version 4.0. Late toxicities were scored according to the RTOG/EORTC toxicity criteria.

Tumor progression was defined as new lesions showing enhancement outside of the radiation field within the first 12 weeks of RT completion, a $25 \%$ increase in the size of one or more measurable lesions or the appearance of new lesions more than 12 weeks after RT completion [15]. Distant metastasis was defined as new lesions that occurred outside the brain. The follow-up duration was defined as the time from the date of surgery to the last date of follow-up for surviving patients or to the date of death. The last follow-up date was $\mathrm{Au}$ gust 1, 2018.

\section{Statistical analysis}

The Kaplan-Meier method was used to evaluate the rates of local recurrence, PFS and OS. The log-rank test and Cox regression method were used for univariate and multivariate analyses, respectively. Statistical analysis was performed with SPSS software (version 20.0; SPSS Inc., Chicago, IL), and $P<0.05$ was considered statistically significant.

\section{Results}

Between February 2012 and February 2018, 80 patients were included in the analysis. The patients' characteristics are listed in Table 1. Fifty patients were men, and 30 patients were women. The median age was 50 years (range, 24 75 years). Forty-four patients received partial resection surgery, and 36 patients received complete resection surgery. Among these patients, 75 were treated with hypofractionated SIB-IMRT and concomitant TMZ, while 5 patients were not treated with concomitant TMZ for personal reasons. Sixty-four patients received adjuvant TMZ, while 16 patients did not receive adjuvant TMZ for economic reasons. The median number of adjuvant chemotherapy cycles was 5 (range, $0 \sim 12$ ).

\section{Efficacy}

After a median follow-up of 16 months (range, 5 72 months), 45 (56.3\%) patients had died, and 49 (64.8\%) patients exhibited tumor progression. The median OS and PFS rates were 21 months $(95 \%$ confidence interval 
Table 1 Demographic and Baseline Clinical Characteristics of Patients

\begin{tabular}{ll}
\hline Variables & Patients N. (N=80) \\
\hline Sex & $50(62.5 \%)$ \\
Male & $30(37.5 \%)$ \\
Female & \\
Age & $50(24-75)$ \\
Median (range) & $39(48.8 \%)$ \\
$<50$ & $41(51.2 \%)$ \\
$\geq 50$ & \\
Extant of surgery & $44(55.0 \%)$ \\
Partial resection & $36(45.0 \%)$ \\
Complete resection & \\
Concurrent TMZ & $75(93.8 \%)$ \\
Yes & $5(6.2 \%)$ \\
No & \\
KPS scores & \\
$90-100$ & $41(51.2 \%)$ \\
80 & $16(20.0 \%)$ \\
$\leq 70$ & $23(28.8 \%)$ \\
TMZ cycles & \\
Median (range) & $5(0-12)$ \\
$<6$ & $42(52.5 \%)$ \\
\hline
\end{tabular}

[CI], 17.5-24.4) and 15 months (95\% CI, 11.0-18.9), respectively. The 1-, 2-, and 3-year rates of PFS among the whole group were 56.0, 27.6, and 19.5\%, respectively (Fig. 2a). The 1-, 2-, 3-, and 5-year rates of OS were 77.6, 41.6, 32.8, and 13.4\%, respectively (Fig. 2b). Age, extent of surgery, KPS scores and the total number of TMZ cycles were significant factors influencing PFS and OS in the univariate analyses (Table 2). In the multivariate analysis, age, extent of surgical resection and the total number of TMZ cycles were significant factors influencing PFS (Fig. 3); age, KPS scores and the total number of TMZ cycles were significant factors influencing OS (Fig. 4).

\section{Patterns of failure}

Tumor progression was detected in 49 (61.3\%) patients, and progression was identified on MRI in 36 of them (Table 3). Sixteen patients had tumor progression within the GTV, 2 patients developed new lesions within CTV1, 1 patient developed new lesions within CTV2, 11 patients developed new lesions outside the radiation field and 6 patients had multicentric progression (both within and outside of the radiation field). The progression sites of 13 patients were unknown because MRI findings were unavailable. The remaining 22 patients were alive with no evidence of progression.

\section{Toxicity}

The most common acute toxicities were nausea, fatigue, headache and hematologic toxicities, which were mainly in grade 1 or 2 and occurred during the concomitant RT and TMZ period. Five patients experienced grade 3 toxicity, including neutropenia (1 patient, $1.3 \%$ ), anemia (2 patients, $2.5 \%$ ), and thrombocytopenia (2 patients, $2.5 \%$ ). No patients experienced grade 4 toxicity. The most common late adverse effects were cognitive disturbances, which occurred in $4(5.0 \%)$ patients. Three patients (3.7\%) developed radionecrosis. Two patients $(2.5 \%)$ presented with progressive headache and dizziness 1 year after RT, and MRI showed increased enhancement. These patients underwent reoperation. The postoperative pathological examination showed only necrotic tissue and no presence of tumor tissue. One patient (1.2\%) was symptom-free, but MRI showed increased enhancement 3 months after treatment; however, there was a
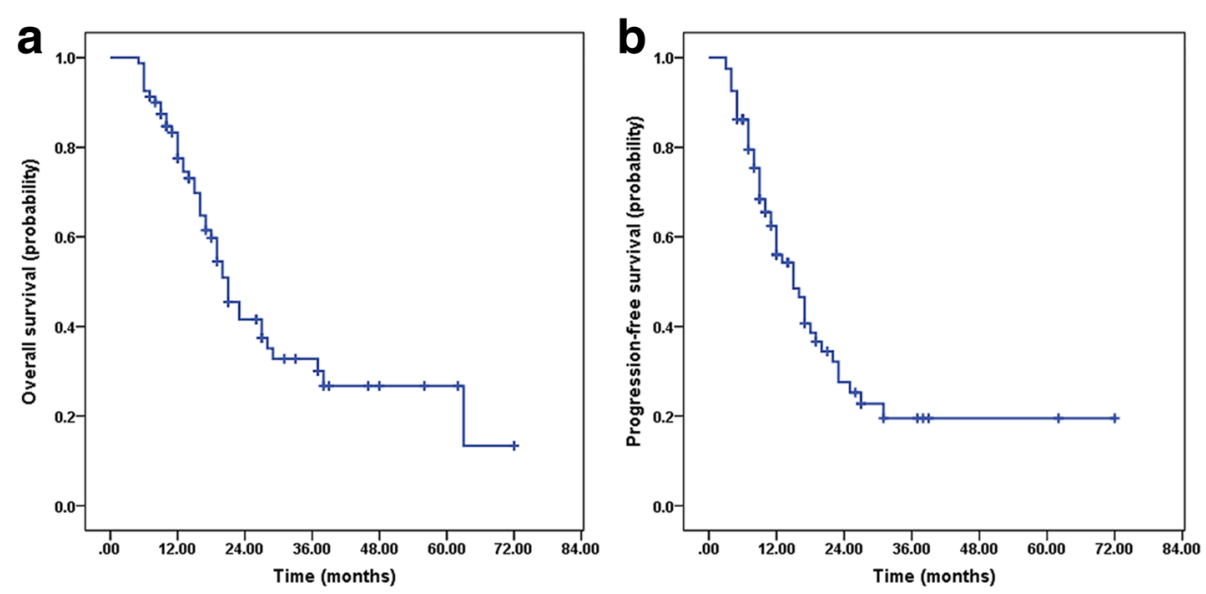

Fig. 2 Kaplan-Meier estimates. a Progression-free survival (PFS). b Overall survival (OS) 
Table 2 Univariate and multivariate analysis of PFS and OS in all patients

\begin{tabular}{|c|c|c|c|c|c|c|c|c|}
\hline \multirow[t]{3}{*}{ Variable } & \multicolumn{2}{|c|}{ Univariate } & \multicolumn{6}{|c|}{ Multivariate } \\
\hline & \multirow{2}{*}{$\begin{array}{l}\text { PFS } \\
P\end{array}$} & \multirow{2}{*}{$\begin{array}{l}\text { OS } \\
P\end{array}$} & \multicolumn{3}{|l|}{$\overline{\mathrm{PFS}}$} & \multicolumn{3}{|l|}{ OS } \\
\hline & & & $\mathrm{HR}$ & $95 \% \mathrm{Cl}$ & $P$ & $\mathrm{HR}$ & $95 \% \mathrm{Cl}$ & $P$ \\
\hline Sex & 0.329 & 0.429 & & & & & & \\
\hline \multicolumn{9}{|l|}{ Male } \\
\hline \multicolumn{9}{|l|}{ Female } \\
\hline Age & $0.026^{\mathrm{a}}$ & $0.022^{a}$ & & & & & & \\
\hline$<50$ & & & $\mathrm{RL}$ & & & $R L$ & & \\
\hline$\geq 50$ & & & 1.894 & $(1.049,3.419)$ & 0.034 & 1.999 & $(1.072,3.727)$ & 0.029 \\
\hline Extent of surgery & $0.003^{\mathrm{a}}$ & $0.040^{\mathrm{a}}$ & & & & & & \\
\hline$P R$ & & & $\mathrm{RL}$ & & & $R L$ & & \\
\hline$C R$ & & & 0.370 & $(0.198,0.692)$ & 0.002 & & & \\
\hline KPS scores & $0.036^{\mathrm{a}}$ & $0.000^{\mathrm{a}}$ & & & & & & \\
\hline $90-100$ & & & & & & $R L$ & & \\
\hline 80 & & & & & & 0.273 & $(0.137,0.542)$ & 0.000 \\
\hline$\leq 70$ & & & & & & 0.265 & $(0.095,0.738)$ & 0.011 \\
\hline Concomitant TMZ & 0.945 & 0.113 & & & & & & \\
\hline \multicolumn{9}{|l|}{ Yes } \\
\hline \multicolumn{9}{|l|}{ No } \\
\hline TMZ cycles & $0.016^{\mathrm{a}}$ & $0.000^{\mathrm{a}}$ & & & & & & \\
\hline$<6$ & & & $\mathrm{RL}$ & & & $R L$ & & \\
\hline$\geq 6$ & & & 0.495 & $(0.275,0.890)$ & 0.019 & 0.246 & $(0.124,0.488)$ & 0.000 \\
\hline
\end{tabular}

Abbreviations: $T M Z$ temozolomide, $P R$ partial resection, $C R$ complete resection, $R L$ referent level

${ }^{\text {a }}$ Significant in univariate analysis

lack of evidence of progression upon MR perfusion and MR spectroscopy, so a diagnosis of clinical radionecrosis was made. All patients completed the planned RT treatment. No treatment-related death occurred.

\section{Discussion}

Previous studies have shown that hypofractionated SIBIMRT combined with TMZ for the treatment of GBM has favorable outcomes on survival [12-14]. However, the larger the dose per fraction delivered, the higher the rate of cerebral necrosis is $[10,16]$. Cho et al. reported that a slightly hypofractionated regimen in association with TMZ chemotherapy had encouraging survival rates without increasing the risk of unacceptable toxicity [12]. In this study, we showed that moderately hypofractionated SIB-IMRT combined with TMZ for the
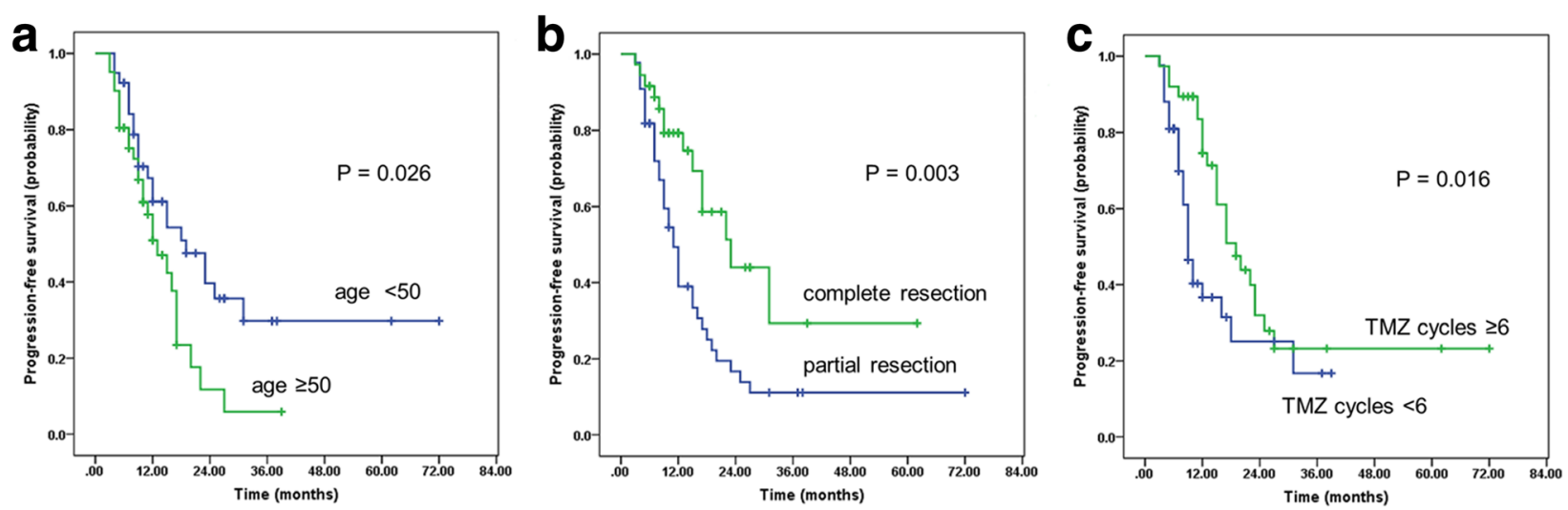

Fig. 3 Kaplan-Meier estimates of progression-free survival (PFS). a PFS for patients of different ages. b PFS for patients who received different extents of surgical resection. $\mathbf{c}$ PFS for patients with different numbers of adjuvant TMZ cycles 

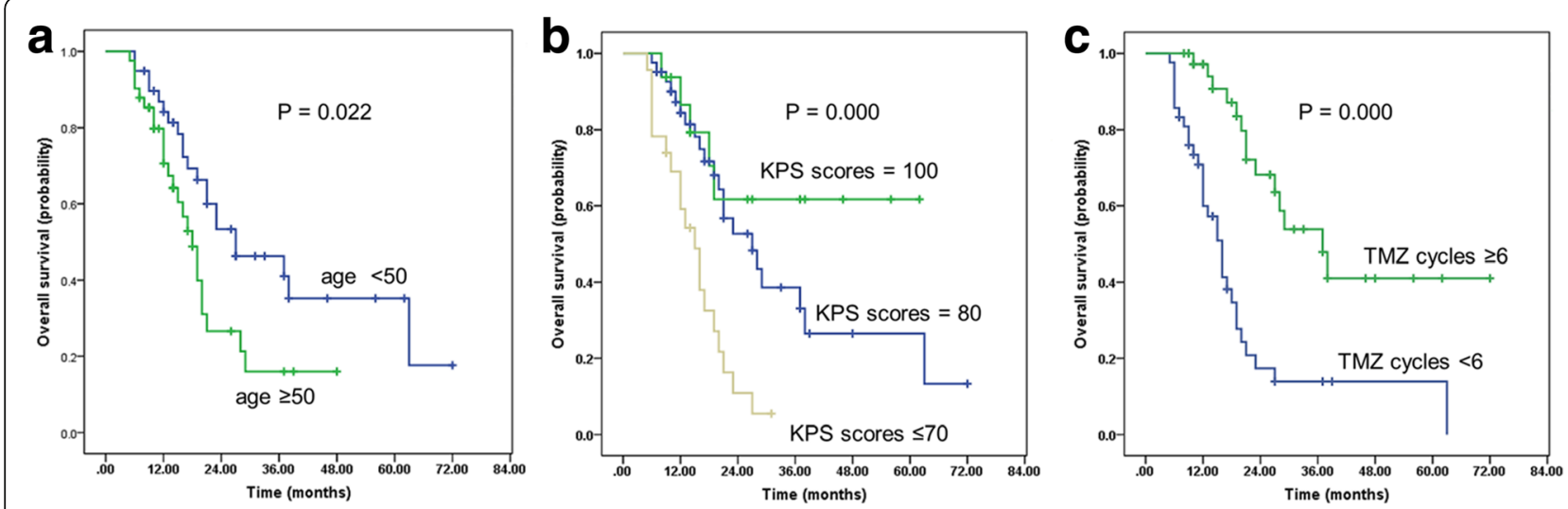

Fig. 4 Kaplan-Meier estimates of overall survival (OS). a OS for patients of different ages; b OS for patients with different KPS scores; c OS for patients with different numbers of adjuvant TMZ cycles

postoperative treatment of GBM had a good outcome, with a median OS of 21 months and a median PFS of 15 months. Sultanem et al. [17] conducted a study to evaluate the efficacy of hypofractionated SIB-IMRT in the treatment of 25 patients with GBM. A total of $60 \mathrm{~Gy}$ over 20 daily fractions of $3 \mathrm{~Gy}$ each were applied to the GTV, and the PTV received a minimum of 40 Gy over 20 fractions of $2 \mathrm{~Gy}$ each at its periphery. The median OS was 9.5 months, and the median PFS was 5.2 months. Acute toxicity occurred in $2(8 \%)$ patients, and no patient developed late toxicity. Panet-Raymond et al. [13] carried out a study to observe the efficacy of SIB-IMRT combined with TMZ in 35 patients with GBM. During a 4-week period, doses of 60 Gy and 40 Gy were delivered in 20 fractions prescribed to the GTV and PTV, respectively. The median survival was 14.4 months, and the median disease-free survival was 7.7 months. The most common acute toxicity was moderate fatigue. No patient developed late toxicity. Mallick et al. [18] compared conventionally fractionated radiation therapy (CRT) and hypofractionated accelerated radiation therapy (HART) in 83 patients with GBM who were between the ages of 16 and 65 years old. The prescribed dose was 50 Gy over 25 fractions to CTV50, followed by a boost of $10 \mathrm{~Gy}$ over 5 fractions to CTV60 in the CRT arm. The prescribed dose was 60 Gy over 20 fractions to CTV60 and 50 Gy over 20 fractions to CTV50 in the HART arm.

Table 3 Patterns of recurrence

\begin{tabular}{ll}
\hline Sites of recurrence & Number of patients \\
\hline Within GTV & 16 \\
Within CTV1 & 2 \\
Within CTV2 & 1 \\
Outside the target volume & 11 \\
Multicentric recurrence & 6 \\
Unknown & 13 \\
\hline
\end{tabular}

The median OS for the entire cohort was 23.4 months, but the median OS was not significantly different between the two arms, with a median OS of 18.07 and 25.18 months $(p=0.3)$. The median PFS for the entire cohort was 13.5 months. One patient (1.2\%) had documented radionecrosis. Scoccianti et al. [19] evaluated the efficacy and toxicity of hypofractionated SIB combined with TMZ in 24 patients with relatively a good prognosis (recursive partitioning analysis classes III and IV). A total dose of 52.5 Gy over 15 fractions and 67.5 Gy over 15 fractions was delivered to the SIB volume. The median OS was 15.1 months, and the median PFS was 8.6 months. One patient $(4.2 \%)$ developed radionecrosis of the brain parenchyma. Although our survival outcome seems to be superior to the results reported by most other studies, it is hard to compare our results with these studies directly because the definition of target volumes and fractionation schedules employed in among studies vary widely. In addition, other studies not only included patients who had received surgical resection but also those who received only biopsy, whereas our study included only patients who had received surgery. Only a well-designed randomized trial can confirm whether our regimen is comparable or superior to the standard treatment regimen.

Age, KPS scores, extent of surgical resection, and the addition of adjuvant chemotherapy have been shown to be significant prognostic factors [20-22]. Our results are similar to those previously reported. Age, KPS scores and the total number of adjuvant TMZ cycles were significant factors influencing $O S$ in the univariate and multivariate analyses. The pairwise comparisons of OS within different KPS scores demonstrated that patients who had a higher KPS score exhibited improved survival. This result agreed with Scoccianti's multicenter study [22] in which 1059 eligible patients were enrolled, and they concluded that patients with higher 
postoperative KPS scores had better survival. Adjuvant chemotherapy is another important prognostic factor. In the present study, we found that patients who received adjuvant TMZ for more than 6 cycles had a median survival of 28 months (95\% CI 20.3-35.7, $p=0.004$ ) compared to 16 months (95\% CI 13.5-18.5, $p=0.004$ ) in patients who received fewer than 6 cycles of adjuvant TMZ. This finding is consistent with that in Darlix's study [23], which showed that prolonged TMZ administration may improve PFS and OS.

The major cause of failure in this study was tumor progression, and no patients had distant metastases. The most common site of recurrence was within the GTV. This finding is in concordance with that observed in other studies [24-26], which suggested that the majority of first GBM relapses following RT with TMZ occurred near the original contrast-enhanced mass. We found that the most common site of recurrence outside the target volume was the corpus callosum and the area close to the lateral ventricle, which occurred in 9 patients. Tumors can reportedly diffuse in white matter along the fiber bundles, such as the longitudinal fasciculi, uncinate fasciculus, visual bundles, corona radiata, etc. [27]. This phenomenon suggests that the current target volume in the area of fiber bundles may not be sufficient. To confirm our results, a randomized phase II trial (chiCTR1800014396) was conducted, and the prospective study will compare the moderately hypofractionated SIB-IMRT regimen with conventionally fractionated RT. The target volume will be delineated according to the patient's neuroanatomy.

It has been reported previously that the acute and late toxicities of SIB-IMRT combined with TMZ in patients with GBM were mild [12-14, 17-19], as was the case in our study. The most common acute toxicities during the period of concomitant RT and TMZ were nausea, fatigue, headache and hematologic toxicities; only 5 patients (6.3\%) experienced grade 3 toxicity, and no patients experienced grade 4 acute toxicities. Three patients (3.7\%) developed radionecrosis. In addition, all patients finished the planned RT. No treatment-related deaths occurred. Therefore, the toxicity of the treatment modality used in our study was mild and tolerable.

The strengths of this study include the relatively large number of patients analyzed. In addition, we adopted moderately hypofractionated RT, which may improve the efficacy of RT while minimizing toxicity. However, the study has some limitations. First, this retrospective study relied heavily on medical records, and some bias exists in the course of data collection. Furthermore, previous studies have shown that O6-methylguanine-DNA-methyltransferase (MGMT) promoter methylation is a significant prognostic biomarker. Unfortunately, because molecular subtyping is not routinely performed in our institution, the MGMT status was not explored.

\section{Conclusions}

Moderately hypofractionated SIB-IMRT combined with TMZ appears to be an effective and safe regimen for postoperative patients with GBM. This novel RT schedule may be an alternative treatment mode for GBM.

\section{Abbreviation}

CTV: Clinical target volume; GBM: Glioblastoma multiforme; GTV: Gross tumor volume; KPS: Karnofsky performance status; MGMT: O6-methylguanine-DNAmethyltransferase; OARs: Organs at risk; OS: Overall survival; PFS: Progressionfree survival; PTV: Planning target volume; RT: Radiotherapy; SIB-

IMRT: Simultaneous integrated boost intensity-modulated radiotherapy;

TMZ: Temozolomide

\section{Acknowledgments}

Not applicable.

\begin{abstract}
Authors' contributions
$\mathrm{ZL}$ and $\mathrm{CL}$ carried out the data analyses, performed the statistical analysis and were major contributors in writing the manuscript. LS and LQ helped with data collection and drafting the manuscript. LW, CG and ZP performed the follow-up; LG designed, coordinated, and supervised the study and critically reviewed and discussed the manuscript. All authors have read and approved the final version of the manuscript.
\end{abstract}

Funding

No funding was received.

\section{Availability of data and materials}

The datasets used and analyzed during the current study are available from the corresponding author on reasonable request.

Ethics approval and consent to participate

This study was approved by the institutional review board and ethics committee of Xinqiao Hospital, Army Medical University, Chongqing, China.

\section{Consent for publication}

Not applicable.

\section{Competing interests}

We declare that we do not have any commercial or associative interests that represent a conflict of interest in connection with the work submitted.

\section{Author details}

${ }^{1}$ Cancer Research Institute of the Chinese People's Liberation Army, Xinqiao Hospital, Army Medical University, Chongqing 400037, China. ${ }^{2}$ Department of neurosurgery, Xinqiao Hospital, Army Medical University, Chongqing 400037, China. ${ }^{3}$ Biobank, Southwest Hospital, Army Medical University, Chongqing 400038, China.

Received: 26 February 2019 Accepted: 24 May 2019

Published online: 13 June 2019

References

1. Stupp R, Hegi ME, Mason WP, et al. Effects of radiotherapy with concomitant and adjuvant temozolomide versus radiotherapy alone on survival in glioblastoma in a randomised phase III study: 5-year analysis of the EORTC-NCIC trial. Lancet Oncol. 2009;10(5):459-66.

2. Chamberlain MC. Radiographic patterns of relapse in glioblastoma. J NeuroOncol. 2011;101(2):319-23.

3. Dobelbower MC, Burnet lii OL, Nordal RA, et al. Patterns of failure for glioblastoma multiforme following concurrent radiation and temozolomide. J Med Imaging Radiat Oncol. 2011;55(1):77-81.

4. McDonald MW, Shu HK, Curran WJ Jr, et al. Pattern of failure after limited radiotherapy and temozolomide for glioblastoma. Int J Radiat Oncol Biol Phys. 2011;79(1):130-6.

5. Minniti G, Amelio D, Amichetti M, et al. Patterns of failure and comparison of different target volume delineations in patients with glioblastoma treated with conformal radiotherapy plus concomitant and adjuvant temozolomide. Radiother Oncol. 2010;97(3):377-81. 
6. Curran WJ Jr, Scott CB, Horton J, et al. Recursive partitioning analysis in three radiation therapy oncology group malignant glioma trials. J Nat Cancer Inst. 1993;85(9):704-10.

7. Nakagawa K, Aoki Y, Fujimaki T, et al. High dose conformal radiotherapy influenced the pattern of failure but did not improve survival in glioblastoma multiforme. Int J Radiat Oncol Biol Phys. 1998;40(5):1141-9.

8. Wang JZ, Li XA. Impact of tumor repopulation on radiotherapy planning. Int J Radiat Oncol Biol Phys. 2005;61(1):220-7.

9. Floyd NS, Woo SY, Teh BS, et al. Hypofractionated intensity-modulated radiotherapy for primary glioblastoma multiforme. Int J Radiat Oncol Biol Phys. 2004;58(3):721-6

10. luchi T, Hatano K, Narita $Y$, et al. Hypofractionated highdose irradiation for the treatment of malignant astrocytomas using simultaneous integrated boost technique by IMRT. Int J Radiat Oncol Biol Phys. 2006; 64(5):1317-24

11. Suzuki M, Nakamatso K, Kanamori S, et al. Feasibility study of the simultaneous integrated boost (SIB) method for malignant gliomas using intensity-modulated radiotherapy (IMRT). Jpn J Clin Oncol. 2003;33(6):271-7.

12. Cho KH, Kim JY, Lee SH, et al. Simultaneous integrated boost intensitymodulated radiotherapy in patients with highgrade gliomas. Int J Radiat Oncol Biol Phys. 2010;78(2):390-7.

13. Panet-Raymond V, Souhami L, Roberge D, et al. Accelerated Hypofractionated intensity-modulated radiotherapy with concurrent and adjuvant Temozolomide for patients with glioblastoma Multiforme: a safety and efficacy analysis. Int J Radiat Oncol Biol Phys. 2009;73(2):473-8.

14. Chen C, Damek D, Gaspar LE, Waziri A, et al. Phase I trial of hypofractionated intensitymodulated radiotherapy with temozolomide chemotherapy for patients with newly diagnosed glioblastoma multiforme. Int J Radiat Oncol Biol Phys. 2011;81(4):1066-74.

15. Wen PY, Macdonald DR, Reardon DA, et al. Updated response assessment criteria for high-grade gliomas: response assessment in neuro-oncology working group. J Clin Oncol. 2010;28(11):1963-72.

16. Yoon SM, Kim JH, Kim SJ, et al. Hypofractionated intensity-modulated radiotherapy using simultaneous integrated boost technique with concurrent and adjuvant temozolomide for glioblastoma. Tumori. 2013; 99(4):480-7.

17. Sultanem K, Patrocinio $H$, Lambert C, et al. The use of hypofractionated intensity-modulated irradiation in the treatment of glioblastoma multiforme: preliminary results of a prospective trial. Int J Radiat Oncol Biol Phys. 2004; 58(1):247-52.

18. Mallick S, Kunhiparambath H, Gupta S, et al. Hypofractionated accelerated radiotherapy (HART) with concurrent and adjuvant temozolomide in newly diagnosed glioblastoma: a phase II randomized trial (HART-GBM trial). J Neuro-Oncol. 2018;140(1):75-82.

19. Scoccianti S, Krengli M, Marrazzo L, et al. Hypofractionated radiotherapy with simultaneous integrated boost (SIB) plus temozolomide in good prognosis patients with glioblastoma: a multicenter phase II study by the brain study Group of the Italian Association of radiation oncology (AIRO). Radiol Med. 2018;123(1):48-62.

20. Lacroix M, Abi-Said D, Fourney DR, et al. A multivariate analysis of 416 patients with glioblastoma multiforme: prognosis, extent of resection, and survival. J Neurosurg. 2001;95(2):190-8.

21. Darefsky AS, King JT Jr, Dubrow R. Adult glioblastoma multiforme survival in the temozolomide era: a population-based analysis of surveillance, epidemiology, and end results registries. Cancer. 2012;118(8):2163-72.

22. Scoccianti S, Magrini SM, Ricardi U, et al. Patterns of care and survival in a retrospective analysis of 1059 patients with glioblastoma multiforme treated between 2002 and 2007: a multicenter study by the central nervous system study Group of Airo (Italian Association of Radiation Oncology). Neurosurgery. 2010;67(2):446-58.

23. Darlix A, Baumann C, Lorgis V, et al. Prolonged administration of adjuvant temozolomide improves survival in adult patients with glioblastoma. Anticancer Res. 2013;33(8):3467-74.

24. Sherriff J, Tamangani J, Senthil L, et al. Patterns of relapse in glioblastoma multiforme following concomitant chemoradiotherapy with temozolomide. Br J Radiol. 2013:86(1022):20120414

25. McDonald MW, Shu HG, Curran WJ, Crocker IR. Pattern of failure after limited margin radiotherapy and temozolomide for glioblastoma. Int J Radiat Oncol Biol Phys. 2011;79(1):130-6.
26. Milano MT, Okunieff $P$, Donatello RS, et al. Patterns and timing of recurrence after temozolomide-based chemoradiation for glioblastoma. Int J Radiat Oncol Biol Phys. 2010;78(4):1147-55.

27. Schiffer D. Neuropathology and imaging: the ways in which glioma spreads and varies in its histological aspect. In: Walker M.D.,Thomas D.G.T., editors. Biology of brain tumour. Boston: Springer; 1986. p. 163-72.

\section{Publisher's Note}

Springer Nature remains neutral with regard to jurisdictional claims in published maps and institutional affiliations.
Ready to submit your research? Choose BMC and benefit from:

- fast, convenient online submission

- thorough peer review by experienced researchers in your field

- rapid publication on acceptance

- support for research data, including large and complex data types

- gold Open Access which fosters wider collaboration and increased citations

- maximum visibility for your research: over $100 \mathrm{M}$ website views per year

At $\mathrm{BMC}$, research is always in progress.

Learn more biomedcentral.com/submissions 